INTERNATIONAL JOURNAL OF SYSTEMATIC BACTERIOLOGY

Vol. 17, No. 2 April 1967

pp. $157-163$

Copyright 1967 by the Iowa State University Press

\title{
USE OF ACTINOPHAGES FOR THE CLASSIFICATION \\ OF ACTINOMYCETALES *
}

\author{
J. L. Rautenstein
}

Institute of Microbiology, USSR Academy of Sciences, Profsojusnaja, Moscow - B133, U.S.S.R.

Phage typing is known to have a wide application in the classification of bacteria, the pathogenic bacteria in particular. High specificity of some bacteriophages makes it possible to differentiate not only the species of bacteria but also the serologically similar variations within the same species. Some countries possess collections of well-tested bacteriophages which facilitate the classification of pathogenic bacteria and the epidemiological analysis. Standard bacteriophages are prepared and sent out by the International Typing Center in London. Bacteriophages have proved to be especially useful for classification of Salmonellae. The technique of typing of the se cultures by means of phages has been fully elucidated.

When the actinophages were discovered the question a rose about their use for actinomyces differentiation. The literary data on this subject are not plentiful. But the results of some authors: Waksman, Reilly et Harris (1947); Welsch et Pinkaers (1957); Bradley et Anderson (1958); J.I. Rautenstein (1957,1960); Nikitina et Backus (1961) and others demonstrated that actinophages can be successfully applied to Actinomyces classification. It was proved that in some cases there exists a doubtless relationship between the susceptibility to definite actinophages and other properties of the culture: e. g. morphology, production of pigments, production of antibiotics, etc. It is known that the cultures of each species are characterized by a number of properties which belong only to this species. The question about the role of each property for species classification is still disputable, but it is beyond doubt that the stable properties are most important and decisive.

* Presented at the "Round Table Conference on Actinomycetes" held by the Subcommittee on Taxonomy of the Actinomycetes of the ICBN, IXth International Congress for

Microbiology, Moscow, July, 1966. 
One of the most stable properties of a culture is its ability to be lysed by a certain phage. Our observations on the sensitivity of Actinomyces to the certain phages active against them showed that actinomycetes did not change their attitude to specific phages after long storage under most variable conditions.

It is known that a culture sensitive to a phage may become resistant to it. This may be the result of mutation or lysogenization by this phage or the related one. But the frequency of appearance of spontaneous phage-resistant mutants is very low (of the order of $10-7-10-10$ ). That is why the appearance in the population of so small an amount of phageresistant mutants cannot influence the results of phage typing. We did not observe any spontaneous phage-resistant mutants in the various species of Actinomyces we studied. However, when the culture is grown for a long time, variants sensitive to the phages to which the original culture was resistant occur more frequently. This phenomenon is observed in the lysogenic cultures which can lose the prophage and become the refore sensitive to this phage. But the cultures cannot acquire new prophages spontaneously without the attack of the exogenous phage.

Consequently, there is no reas on to believe that the spontaneous formation of phage-resistant mutants may lead to erroneous conclusions in phage-typing.

The investigations carried out in recent years showed that lysogeny is widespread among the actinomycetes and it is no exaggeration to say that the majority of actinomycetes are lysogenic and, probably, polylysogenic. Lysogenic cultures are, as a rule, resistant to the phages they contain. That is why when the phages isolated from lysogenic cultures are used for phage typing there is a serious danger that the greate $r$ part of the strains in the given species will not be identified. But in some cases this difficulty can also be overcome. This question will be dealt with below.

It is evident that for successful classification of actinomycetes by means of actinophages it is necessary to have specific actinophages. Actinophages are widespread in soils. But they are polyphages, $\underline{i}$ e. actinophages with a wide lytic spectrum. Such actinophages are apparently useless for classification of actinomycetes into species and varieties.

It is possible that some polyphages may prove useful for studying some questions of phylogeny of actinomycetes and their division into larger taxonomic units. Tnus, according to data by Bradley and Anderson (1958), some of the actino- 
phages they isolated lysed both Nocardia and Streptomyces but did not lyse the cultures of Streptosporangium, Actinoplanes and Micromonospora. The above authors conclude that this fact favours a phylogenetic relationship between the genera Nocardia and Streptomyces and the advisability of their placing them in one family.

But the most difficult question in the actinomycete taxonomy is that of the species classification. It is especially true for the classification of morphologically and physiologically related cultures. Hence, it is very important to isolate actinophages with limited lytic spectra which are specific for the related groups of microorganisms and, first and foremost, for the cultures of the definite species. The data published on this question show that a number of actinophages with the limited lytic spectra have been isolated, but only rarely from soils. The most promising source for the specific phages is lysogenic cultures. Almost all actinophages described in the literature as specific were isolated from lysogenic cultures and very often from those used in the production of antibiotics. The analysis of literary data and our own observations confirm the fact that in all these cases the re appear virulent mutants of temperate phages of lysogenic cultures. This is the result of mutation of the temperate phage acquiring the ability to lyse the host culture. I'd like to give some examples. Welsch (1957) described some actinophages active against $\underline{S}$. griseus $W$. and H. (A. streptomycini Kras.). But the phage he isolated from a commercial culture was proved most specific for cultures of this species producing streptomycin. According to the data of Nikitina and Korenyako (1958), the actinophage we isolated from the lysed commercial culture of A. streptomycini Kras. turned out to be rather specific. Out of the cultures belonging to the Globisporus group, it could lyse only the cultures belonging to species $\underline{A}$. streptomycini producing streptomycin.

Weindling et al. (1961) isolated actinophages specific for

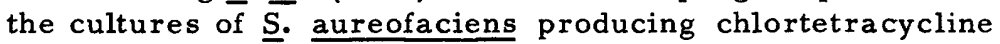
froma lysed commercial culture in the course of chlortetracycline production. A number of cultures originally deter-

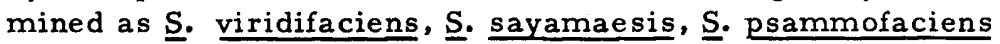
(S. feofaciens) were sensitive to this phage and were proved to belong to $\underline{S}$. aureofaciens. Out of 25 strains of $\underline{S}$. aureofaciens producing chlortetracycline 18 were proved sensitive to the phage in question; the remaining 7 cultures were resistant to this phage due to their lysogenic state. We iso- 
lated actinophages highly specific for the cultures of $\underline{A}$. aureofaciens producing chlortetracycline from the lysed commercial culture. We described earlier the morphology of these actinophages (Rautenstein and Mach 1960).

Actinophages specific for the cultures of $\underline{S}$. mediterranei producing rifamycin were isolated by Thiemann et al. (1962) from a lysed culture.

Hengeller et al. (1965) observed the phage lysis of the commercial culture of Streptomyces sp. 23 producing novobiomycin and isolated a specific actinophage. We showed, together with Klepitova, Zhunaeva and Panichkova(1965), that the culture $\underline{A}$. spheroides 35 is lysogenic and contains a very specific actinophage.

We, together with Retinskaya (1960), isolated an actinophage highly specific for the cultures A. erythreus producing erythromycin from the lysogenic culture $A$. erythreus. We also isolated a very specific actinophage from the lysogenic culture of A. levoris. According to our data (1957), and also those by Nikitina and Korenyako (1958) and Kusnetsov (1960), this phage was very useful for differentiation of cultures producing polyene antibiotics.

Phages specific for cultures of A. olivaceus were isolated from the lysogenic cultures of this species by Khavina and Rautenstein (1950).

All the above data, which are far from being complete, testify that the specific actinophages isolated from lysogenic cultures can be successfully applied to Actinomyces differentiation. In a number of cases actinophages proved very useful for accurate identification of cultures.

As was mentioned above, it is a matter of some difficulty to use temperate phages isolated from lysogenic cultures for the identification of the cultures belonging to the same species. Such cultures are resistant to the phages due to their lysogenic state. This difficulty may be overcome by means of experimentally obtaining from the temperate phages virulent mutants with a wider lytic spectrum. This is achieved by the action of mutagenic agents, by selection of more virulent mutants which appear in the population, or by changing the phage under the influence of the host culture, i. e. the culture used for reproduction.

Our experimental data show that all the above ways are possible. There are some phages, however, for which the virulent mutants are very difficult to obtain.

The following data on the variability of the actinophage specific for cultures of $\underline{A}$. streptomycini (‥ griseus) may 
be cited as an example of successful acquisition of virulent mutants. We have three types of this phage. The first type was isolated from a commercial culture which was lysed during the fermentation; the second type was obtained from the first type grown on a culture of $\underline{A}$. streptomycini resistant to it; the third type was obtained as a result of growing the phages of type two on the cultures resistant to phages of types one and two. Table 1 shows the results of the experiments studying the activity of the above phages on 214 streptomycin-producing cultures of the species $\underline{A}$. streptomycini Kras. isolated from various sources.

\section{Table 1}

\begin{tabular}{lllll}
\hline & \multicolumn{4}{c}{ Cultures sensitive to } \\
\cline { 2 - 5 } $\begin{array}{l}\text { Total No. } \\
\text { cultures } \\
\text { tested }\end{array}$ & $\begin{array}{l}\text { Actinophages A. } \\
\text { Ist type } \text { IInd type }\end{array}$ & $\begin{array}{l}\text { Actinophage } \\
\text { specific for } \\
\text { And type }\end{array}$ & $\begin{array}{l}\text { A. levoris } \\
214\end{array}$ \\
\hline
\end{tabular}

As is seen from the table, out of 214 cultures of the species A. streptomycini of various origin no culture was resistant to the IIIrd type phage. There is reason to believe that in the natural condition the cultures resistant to this phage are extremely rare or do not exist at all. According to the data by Prauser and Kohler (1965) the IIIrd type actinophage of $\underline{A}$. Streptomycini was useful in the differentiation of the cultures belonging to this species.

We also obtained virulent mutants of phages of $\underline{A}$. erythreus, $A$. olivaceus, $\underline{A}$. aureofaciens and others capable of lysing respective lysogenic cultures.

We observed in actinophages of the culture $\underline{A}$. levoris (1959) and A. fradiae and others the considerable alteration in the lytic spectrum resulting from the influence of the host culture. It was stated, therefore, that as a result of reproduction in some cultures there appear phages with a wider spectrum; in other cultures - with a very limited spectrum. So it is possible to obtain phages with a very wide spectrum or to make a mixture of various variations of the given phage which will be active against all the cultures of the given species. 
Virulent mutants are especially expedient for phage typing as they form a very clear lysed zone. There are some actinophages isolated from lysogenic cultures which are capable of lysing cultures belonging to other species. This is true of the actinophage attacking the streptomycin produce $r$ which is also active against some strains of $\mathrm{A}$. olivaceus, A. viridis and others. This phenomenon deserves further study, but in our opinion it does not reduce the value of the above phage for the actinomycete classification.

Unfortunately, at present the number of specific actinophages is not great. We have in our collection specific actinophages for the following species, with virulent mutants obtained from the majority of them:
A. aureofaciens
A. levoris
A. erythraeus
A. fradiae
A. olivaceus
A. viridochromogenes

A. gobitricini

A. venezuelae

A. $\underline{\text { lavendulae }}$

A. streptomycini Kras. (S. griseus)

A. spheroides

Proactinomyces fructiferi

One of the main tasks of the workers in actinomycete taxonomy using typing phages is to facilitate isolation of specific actinophages and their testing on a vast scale. Scientists of a number of countries should take part in this work. It is necessary to organize exchanges of actinophages for testing with the aim of making a collection of typing phages and corresponding host cultures.

The question about the possibility of classification of Actinomycetes by means of their prophages has not been studied. Such methods may be very useful for the establishment of relationships among the cultures.

In conclusion I'd like to dwell on the methods applied in the phage typing of actinomycetes.

Phage lysates used for typing may contain lytic substances of various natures (lysines, antibiotics and various bacteriocines) whose action may be mistaken for the phage lytic activity. This difficulty may be overcome by the reproducibility tests as well as phage lysate dilutions with the removed bacteriocine action (the method of standard dilutions).

The typing actinophages should be reproduced only on the definite cultures. The best method of determining the culture sensitivity to the phage is to place a drop of the phage on the surface of the freshly prepared growth in a Petri dish. 
Sparse negative colonies (plaques) on the site where the phage was placed do not signify that the test culture is sensitive to the phage if there is a complete lysis on the control culture. The medium has a great effect on the phage lytic activity-hence the necessity of choosing the optimalmedium for typing phages. 